\title{
A Study on the Proposed Korean Digital Signature Algorithm ${ }^{\star}$
}

\author{
Chae Hoon $\operatorname{Lim}^{1}$ and Pil Joong Lee ${ }^{2}$ \\ 1 Future Systems, Inc., Seoul, Korea \\ email: chlim@future.co.kr \\ 2 Dept. Electronic and Electrical Eng., POSTECH, Pohang, Korea \\ email: pj1@postech.ac.kr
}

\begin{abstract}
A digital signature scheme is one of essential cryptographic primitives for secure transactions over open networks. Korean cryptographic community, in association with government-supported agencies, has made a continuous effort over past three years to develop our own signature standard. The outcome of this long effort is the signature algorithm called KCDSA, which is now at the final stage of standardization process and will be published as one of KICS (Korean Information and Communication Standards). This paper describes the proposed signature algorithm and discusses its security and efficiency aspects.
\end{abstract}

\section{Introduction}

The digital signature technique, a technique for signing and verifying digital documents in an unforgeable way, is essential for secure transactions over open networks. Digital signatures can be used in a variety of applications to ensure the integrity of data exchanged or stored and to prove to the recipient the originator's identity.

A group of Korean cryptographers, in association with government-supported agencies, has been developing a candidate algorithm for Korean digital signature standard, which is named KCDSA temporarily (standing for Korean Certificatebased Digital Signature Algorithm). As a result of such effort over three years, a final algorithm has been established and is now being standardized by the Korean Government. This signature algorithm, once standardized, is hopefully to be widely supported in commercial security products by Korean industries and possibly by the Government. In addition, a standard hash algorithm, developed for use with KCDSA, is also under standardization process.

\footnotetext{
* KCDSA was developed by a task force team consisting of Sang Jae Moon (Kyung Pook Univ.), Dong Ho Won (Sung Gyun Kwan Univ.), Sung Jun Park (KISA), Chung Ryong Jang (Kyung Dong Univ.), Shin Gak Kang (ETRI), Eun Jeong Lee (POSTECH), Sang Bae Park (IDIS), Chul Kim (Kwang Woon Univ.), Kyung Seok Lee (KIET), Jae Hyun Baek (ADD), Jong Tae Shin (KISA), etc., and the present authors, under the financial support of ETRI (Electronics and Telecommunications Research Institute) and KISA (Korea Information Security Agency).
} 
The security of most signature schemes widely used in practice is based on two difficult problems: the problem of factoring integers (e.g., RSA [16]) and the problem of finding discrete logarithms over finite fields (e.g., Elgamal [5]). The RSA scheme is used in many applications as a de facto standard. On the other hand, two variants of the Elgamal scheme have been standardized in U.S.A as digital signature standard (DSS) [19] and in Russia as GOST 34.10 (see [10]). KCDSA is also a Elgamal-type signature scheme. There have been a lot of discussions on whether our national standard should be either of RSA type or of Elgamal type. There also has been some controversy on establishing a new standard other than the widely used schemes such as RSA and DSA. Putting aside the behind story, we concluded to design our own signature scheme and KCDSA is the outcome. KCDSA is designed by incorporating several features from the recent cryptographic research and thus is believed to be secure and robust.

In this paper we describe the proposed standard for KCDSA and discuss security and efficiency aspects considered during the design process. Throughout this paper we will use the following symbols and notation:

- $a \oplus b$ : exclusive-or of two bit strings $a$ and $b$.

- $a \| b$ : concatenation of two bit strings $a$ and $b$.

$-\mathbf{Z}_{n}=\{0,1, \cdots, n-1\}$ and $\mathbf{Z}_{n}^{*}=\{x \mid 1 \leq x \leq n-1 \& \operatorname{gcd}(x, n)=1\}$.

- $|A|$ denotes the bit-length of $A$ for integer $A$ and the cardinality of $A$ for set A.

$-k \epsilon_{r} S$ denote that $k$ is chosen at random over the set $S$.

This paper is organized as follows. We describe KCDSA parameters in Section 2 and the detailed signature algorithm in Section 3. The security and efficiency aspects of KCDSA are discussed in Sections 4 and 5, respectively. In Section 6 we briefly describe an elliptic curve variant of KCDSA and finally we conclude in Section 7.

\section{KCDSA Parameters}

KCDSA parameters can be divided into domain parameters and user parameters. By domain we mean a group of users who shares the same public parameters (domain parameters). Domain may consist of a single user if the user uses its own public parameters. User parameters denote parameters which are specific to each user and cannot be shared with others. These parameters must be established before normal use of digital signatures by some trusted authorities and/or by users. KCDSA makes use of the following domain and user parameters (see Appendix for a procedure that can be used to generate domain parameters):

Domain Parameters: $p, q, g$ such that

- $p$ : a large prime such that $L_{p}=|p|=512+256 i$ for $i=0,1, \cdots, 6$. That is, the bit-length of $p$ can vary from 512 bits to 2048 bits with increment by a multiple of 256 bits. 
- $q$ : a prime factor of $p-1$ such that $L_{q}=|q|=128+32 j$ for $j=0,1, \cdots, 4$. That is, the bit-length of $q$ can vary from 128 bits to 256 bits with increment by a multiple of 32 bits. Further, it is required that $(p-1) / 2 q$ should be a prime or at least all its prime factors should be greater than $q .1$

$-g$ : a base element of order $q \bmod$ p, i.e., $g \neq 1$ and $g^{q}=1 \bmod p$.

\section{User Parameters: $x, y, z$ such that}

- $x$ : signer's private signature key such that $x \in_{r} \mathbf{Z}_{q}$.

$-y$ : signer's public verification key computed by $y=g^{x^{-1}} \bmod p$, where $x^{-1}$ denotes the multiplicative inverse of $x \bmod q \bigsqcup^{2}$

- $z$ : a hash-value of Cert_Data, i.e., $z=h($ Cert_Data $)$. Here Cert_Data denotes the signer's certification data, which should contain at least Signer's distinguished identifier, public key $Y$ and the domain parameters $\{p, q, g\}$.

KCDSA is a signature algorithm in which the public key is validated by means of a certificate issued by some trusted authority. The X.509-based certificate may be used for this purpose. In this case, the Cert_Data can be simply the formatted certification data defined by X.509.

KCDSA also requires a collision-resistant hash function which produces $L_{q^{-}}$ bit outputs. Since $q$ can vary in size from 128 bits to 256 bits with increment by a multiple of 32 bits, we need a family of hash functions or a hash function which can produce variable length outputs up to 256 bits. Currently standardization is being processed for a hash algorithm with 160-bit outputs called HAS-160. Hash functions for the other sizes of $q$ are left as a future work.

\section{The Signature Algorithm}

\subsection{Signature Generation}

The signer can generate a signature $\{r \| s\}$ for a message $m$ as follows:

1. randomly picks an integer $k$ in $\mathbf{Z}_{q}^{*}$ and computes $w=g^{k} \bmod p$,

2. computes the first part $r$ of the signature as $r=h(w)$,

3. computes $e=r \oplus h(z \| m) \bmod q$, and

4. computes the second part $s$ of the signature as $s=x(k-e) \bmod q$.

${ }^{1}$ This restriction on the size of prime factors of $(p-1) / 2 q$ is to take precautions against possible attacks using small order subgroups of $\mathbf{Z}_{p}^{*}$ in various applications of KCDSA (see 8] for details).

${ }^{2}$ Notice that there is essentially no difference in the signature algorithm if the secretpublic key pair is represented by $\left\{x^{-1} \bmod q, y=g^{x} \bmod p\right\}$. We simply adopted the above notation to clarify (to the public unaware of cryptography) that we only need $x$ for a signing purpose. This kind of key pair may be undesirable if the same key is to be used for other purposes as well (e.g., key exchange or entity authentication). However, it is a common practice in cryptographic protocol designs that the same key should not be used for different purposes. 
The computation of $w$ is the most time-consuming operation in the signing process. However, since the first two steps can be performed independent of a specific message to be signed, we may precompute and securely store the pair $\{r, k\}$ for fast on-line signature generation. The above signing process can be described in brief by the following two equations:

$$
\begin{aligned}
& r=h\left(g^{k} \bmod p\right) \text { with } k \in_{r} \mathbf{Z}_{q}^{*}, \\
& s=x(k-r \oplus h(z \| m)) \bmod q .
\end{aligned}
$$

\subsection{Signature Verification}

On receiving $\{m\|r\| s\}$, the verifier can check the validity of the signature as follows:

1. first checks the validity of the signer's certificate, extracts the signer's certification data Cert_Data from the certificate and computes the hash value $z=h($ Cert_Data $)$ [3

2. checks the size of $r$ and $s: 0<r<2^{|q|}, 0<s<q$,

3. computes $e=r \oplus h(z \| m) \bmod q$,

4. computes $w^{\prime}=y^{s} g^{e} \bmod p$ and

5. finally checks that $r=h\left(w^{\prime}\right)$.

The pair $\{r \| s\}$ is a valid signature for $m$ only if all the checks succeed. The above verifying process can be described in brief by the following equations:

$$
\begin{aligned}
& e=r \oplus h(z \| m), \\
& r=h\left(y^{s} g^{e} \bmod p\right) ?
\end{aligned}
$$

For comparison, we summarized three signature standards, DSA, GOST and KCDSA, in Table 1 .

\section{Security Considerations}

\subsection{Security Proof under Random Oracle Model}

Recently two variants of ElGamal-like signature schemes have been proven secure against adaptive attacks for existential forgery under the random oracle model [3], where the hash function is replaced with an oracle producing a random value for each new query. In the first variant, $h(m)$ is replaced with $h(m \| r)$ as in the Schnorr signature scheme. This variant was proven secure by Pointcheval and Stern [13] at Eurocrypt'96. The other variant is due to Brickell [4] at Crypto'96,

\footnotetext{
${ }^{3}$ Note that a certificate corresponds to a trusted authority's signature for the formatted data containing all information required to bind the public key and related parameters/attributes to the key owner's identity. Therefore, the computation of $z$ can be in fact part of the certificate validation process by taking Cert_Data as the formatted data to be signed.
} 


\begin{tabular}{|c||c|c|}
\hline Schemes & $|p|$ & $|q|$ \\
\hline \hline DSA & $512+64 i(i=0,1, \cdots, 8)$ & 160 \\
\hline GOST & 512 or 1024 & 256 \\
\hline KCDSA & $512+256 i(i=0,1, \cdots, 6)$ & $128+32 i(i=0,1, \cdots, 4)$ \\
\hline
\end{tabular}

\begin{tabular}{|c|c|c|}
\hline Schemes & Signature Generation & Signature Verification \\
\hline \multirow[b]{2}{*}{$\begin{array}{c}\text { DSA } \\
19\end{array}$} & private Key $: x \in_{r} \mathbf{Z}_{q}$ & public key : $y=g^{x} \bmod p$ \\
\hline & $\begin{array}{l}k \in_{r} \mathbf{Z}_{q}^{*} \\
r=\left(g^{k} \bmod p\right) \bmod q \\
s=k^{-1}(r x+h(m)) \bmod q\end{array}$ & $\left(y^{s^{-1} r} g^{s^{-1} h(m)} \bmod p\right) \bmod q=r ?$ \\
\hline $\begin{array}{c}\text { GOST } \\
10\end{array}$ & $\begin{array}{l}k \in_{r} \mathbf{Z}_{q} \\
r=\left(g^{k} \bmod p\right) \bmod q \\
s=r x+k h(m) \bmod q\end{array}$ & $\left(y^{-r h(m)^{-1}} g^{s h(m)^{-1}} \bmod p\right) \bmod q=r ?$ \\
\hline \multirow[b]{2}{*}{ KCDSA } & private Key $: x \in_{r} \mathbf{Z}_{q}$ & public key $: y=g^{x^{-1}} \bmod p$ \\
\hline & $\begin{array}{l}k \in_{r} \mathbf{Z}_{q}^{*} \\
r=h\left(g^{k} \bmod p\right) \\
s=x(k-r \oplus h(z \| m)) \bmod q\end{array}$ & $h\left(y^{s} g^{r \oplus h(z \| m)} \bmod p\right)=r ?$ \\
\hline
\end{tabular}

Table 1. Comparison of DSA, GOST and KCDSA

where he claimed that the variant of DSA with $r=\left(g^{k} \bmod p\right) \bmod q$ replaced by $r=h\left(g^{k} \bmod p\right)$ is also secure in the random oracle model (see [14 for its proof by Pointcheval and Vaudenay). We followed the latter approach to ensure the security of the overall design of KCDSA. From the proof under the random oracle model we can be assured that KCDSA will be secure provided that the hash function used has no weakness.

\subsection{Security against Parameter Manipulation}

There have been published a lot of weaknesses in the design of discrete logbased schemes due to the use of unsafe parameters (later shown insecure) (e.g., see $[122,1,8]$ ). Note that generating public parameters at random so that they do not have any specific structure is very important for security, even with a provably secure scheme (compare the results from [2] and [13]. see also [17]). KCDSA is designed to be secure against all these potential weaknesses. The (proposed) standard recommend to use the strongest form of primes 8], i.e., primes $p, q$ such that $(p-1) / 2 q$ is also a prime or at least its prime factors are all greater than $q$. It also specifies a procedure that can be used for generation of such primes (see Appendix A). The certificate produced by this procedure can be used to verify proper generation of the parameters. Considering current algorithms and technology for finding discrete logarithms (see [11]), we recommend to use a modulus $p$ of size 1024 bits and an auxiliary prime $q$ of 160 bits for moderate security in most applications. 
The use of the parameter $z=h($ Cert_Data $)$ as a prefix message for hashing provides several advantages without much increase of computational/operational overheads 4

It effectively prevents possible manipulations during parameter generation, such as hidden collisions in DSS [18, since Cert_Data contains $p, q, g$ and $y$. In addition, the use of $z$ restricts the collision search in the hash function to a specific signer, since each signer uses his/her own prefix $z$ to produce a hash code for his/her message. To see its usefulness, suppose that in the case of using the usual hash code $h(m)$ a collision is found for a specific pair of messages. Also suppose that one message out of the pair is a comfortable message that anyone can sign without reluctance. Then the collision can be used to any user to claim that the signature is for the harmful message. Realization of this scenario may be catastrophic, for example, if there exists some powerful organization willing to invest a huge amount of money to find collisions (the organization might find some unpublished weakness in the hash function which can substantially reduce the time for exhaustive search). Our new hash mode with a user-specific prefix can effectively thwart such a trial of total forgery unless a serious weakness is found for the hash function.

\section{$5 \quad$ Efficiency Considerations}

KCDSA is designed to avoid the evaluation of multiplicative inverses in normal use. It is only needed at the time of key pair generation. For comparison, in DSA a multiplicative inverse $\bmod q$ needs to be evaluated each time a signature is generated or verified and in GOST each time a signature is verified (see Table 1). Evaluating an inverse $\bmod q$ would take very little portion in the overall workload of signing/verifying on most general purpose computers. However, it may be quite expensive in a limited computing environment such as smart cards (see [15] for various comments on DSS including debates on the use of inverse). On the other hand, KCDSA needs one more call for a hash function to digest a message of length $|p|$ during both the signature generation and the verification process. This will not cost much in any environment.

We have implemented various signature schemes in the $\mathrm{C}$ language with inline assembly [9] and measured their timings on $90 \mathrm{MHz}$ Pentium and 200 $\mathrm{MHz}$ Pentium Pro. The result is shown in Table 2 As can be expected, KCDSA and DSA show almost the same performance figures, but GOST runs about $63 \%\left(\approx \frac{160}{256}\right)$ slower than KCDSA/DSA since it uses a 256-bit prime $q$. For comparison, we also measured the speed of RSA for the same size of modulus.

\footnotetext{
${ }^{4}$ In the present standard the hashed cert. data $z$ is used as part of message (i.e., $z \| m$ is treated as a message to be signed). However, it may be more desirable to separate $z$ from the message to be signed. For example, we may use $z$ itself as a user-specific IV or complete $z$ into one block by zero-padding and use $h(z \|$ pad $)$ as a user-specific IV. These variants will be further discussed in the next revision.

${ }^{5}$ We used SHA-1 for hashing with a very short message in all the signature schemes. Multiplicative inverses were computed using an extended Euclidean algorithm.
} 
Note that signature generation can be substantially speeded up in both RSA and ElGamal-type schemes: We can use the Chinese Remainder Theorem to speed up RSA signature generation and the precomputation technique [7] to speed up signature generation in ElGamal-type schemes. These performance figures are also shown after ' $/$ ' in Sign columns. The table shows that KCDSA/DSA can sign about 6 to 10 times faster than RSA, while RSA can verify about 12 to 13 times faster than KCDSA/DSA (RSA verification key: $e=2^{16}+1$ ).

\begin{tabular}{|c||c||c|c||c|c|}
\hline \multicolumn{1}{|c||}{} & \multicolumn{2}{c||}{} & \multicolumn{2}{c||}{ Pentium/90 } & Pentium Pro/200 \\
\hline Algorithm & Lang. & Sign & Verify & Sign & Verify \\
\hline \hline \multirow{3}{*}{$\begin{array}{c}\text { DSA } \\
|q|=160)\end{array}$} & $\mathrm{C}$ & $289 / 57.8^{\circ}$ & 359 & $95.0 / 18.9$ & 117 \\
\cline { 2 - 6 } & $\mathrm{D}$ & $148 / 29.8$ & 182 & $47.3 / 9.7$ & 58.0 \\
\cline { 2 - 6 } & $\mathrm{A}$ & $64.0 / 13.7$ & 79.1 & $17.5 / 3.9$ & 21.7 \\
\hline \hline \multirow{4}{*}{$\begin{array}{c}\text { GOST } \\
(|q|=256)\end{array}$} & $\mathrm{C}$ & $457 / 87.8$ & 559 & $147 / 28.0$ & 181 \\
\cline { 2 - 6 } & $\mathrm{D}$ & $236 / 44.3$ & 287 & $73.4 / 14.0$ & 92.3 \\
\cline { 2 - 6 } & $\mathrm{A}$ & $105 / 19.1$ & 125 & $27.2 / 5.2$ & 35.3 \\
\hline \hline \multirow{3}{*}{$(|q|=160)$} & $\mathrm{C}$ & $287 / 56.2$ & 359 & $93.3 / 18.0$ & 116 \\
\cline { 2 - 6 } & $\mathrm{D}$ & $145 / 28.0$ & 185 & $46.4 / 9.0$ & 57.4 \\
\cline { 2 - 6 } & $\mathrm{A}$ & $62.8 / 12.4$ & 77.7 & $17.0 / 3.3$ & 20.9 \\
\hline \hline \multirow{3}{*}{$\begin{array}{c}\text { RSA } \\
\left(e 2^{16}+1\right)\end{array}$} & $\mathrm{C}$ & $1730 / 502^{*}$ & 25.8 & $568 / 163$ & 8.6 \\
\cline { 2 - 6 } & $\mathrm{D}$ & $878 / 254$ & 15.8 & $279 / 83.5$ & 5.3 \\
\cline { 2 - 6 } & $\mathrm{A}$ & $378 / 114$ & 6.0 & $103 / 33.1$ & 1.7 \\
\hline
\end{tabular}

Notes :

$\mathrm{C}=\mathrm{C}$ only,

$\mathrm{D}=\mathrm{C}$ with double digit option (_-int64) provided by MSVC,

$\mathrm{A}=\mathrm{C}$ with partial inline assembly.

* used CRT for signature generation.

○ used a precomputation table of 32 KBytes $(6 \times 4$ config., see [7]).

Table 2. Speed of various signature schemes for 1024-bit moduli (in msec)

\section{Elliptic Curve KCDSA}

Much attention has been paid to elliptic curve cryptosystems in recent years, due to their stronger security and higher speed with smaller key size. An elliptic curve variant of KCDSA (EC-KCDSA for short) was not considered during the standardization process. However, we have recently worked on an alternative implementation of KCDSA over elliptic curves and completed a high-level specification of EC-KCDSA. The following brief description on EC-KCDSA is expected to be included in the next revision or as an addendum.

Let $E$ be an elliptic curve over a finite field and $\#(E)$ be the order of $E$ (the total number of points on $E$ ). The curve $E$ should be chosen so that $\#(E)$ is 
divided by a prime $q$ of size $L_{q}$ bits. Domain parameters consist of the description of the elliptic curve $E$, the prime $q$ and a point $G=\left(g_{x}, g_{y}\right)$ over $E$ generating a cyclic group of prime order $q .6$ As user parameters, each signer picks at random a private signature key $x$ over $\mathbf{Z}_{q}^{*}$ and computes the corresponding public key $Y$ as $Y=\bar{x} G$ over $E$, where $\bar{x}=x^{-1} \bmod q$. The hashed certification data $z$ and the hash function $h$ are the same as before. Finally, for simplicity we write $h(W)$ for an elliptic curve point $W=\left(w_{x}, w_{y}\right)$ to denote $h\left(w_{x} \| w_{y}\right)$. Note here that the two coordinates $w_{x}, w_{y}$ are treated as bit strings and thus they are simply concatenated (without conversion from elliptic curve point to integer) and hashed.

The signing and verifying processes of EC-KCDSA are almost the same as those of KCDSA, except for the change of group operations. That is, the underlying group is changed from the multiplicative group of a prime field into the additive group of elliptic curve points. The signature for message $m$ consists of two integers $r, s$ of size $|q|$ generated by

$$
\begin{aligned}
& r=h(k G) \text { with } k \in_{r} \mathbf{Z}_{q}^{*}, \\
& s=x(k-r \oplus h(z \| m)) \bmod q,
\end{aligned}
$$

where the computation of $r$ consists of computing $W=k G$ over $E$ and then hashing the point $W$.

To verify the signature $\{m\|r\| s\}$, a verifier first performs the required checks on the certificate and the size of signature components as in KCDSA (see steps 1 and 2 in Sect.3.2). The verifier then recovers the point $W$ using the received signature and checks the equality $r=h(W)=h\left(w_{x} \| w_{y}\right)$. That is, the verifying process can be described in brief by

$$
\begin{aligned}
& e=r \oplus h(z \| m) \bmod q, \\
& r=h(s Y+e G) ?
\end{aligned}
$$

In general, the security of EC-KCDSA will be stronger than KCDSA if both use the same size of $q$. However, more detailed security and efficiency analyses should be carried out after complete specification on various parameters.

\section{Conclusion}

We described the proposed digital signature standard for Korean community and discussed its security and efficiency. The presented algorithm is now close to publication as one of Korean Information and Communication Standards and hopefully to be widely used in security products by Korean industries and Government. We hope this publication to stimulate further investigation on its security and development of various useful applications based on it.

\footnotetext{
${ }^{6}$ According to [8], the effective key length can be reduced from $|q|$ to $2|q|-|\#(E)|$ bits in some applications of signature schemes. Therefore, considering wide applications of signature schemes, we strongly recommend that an elliptic curve should be chosen so that $\#(E)$ has as small prime factors as possible. Ideally, $|q|=|\#(E)|$.
} 


\section{Acknowledgments}

We are very grateful to Hyo Sun Hwang (Future Systems, Inc.) for his time and effort in implementing various signature schemes for speed comparison.

\section{References}

1. R.Anderson and S.Vaudenay, Minding your $p$ 's and q's, In Advances in Cryptology - ASIACRYPT'96, LNCS 1163, Springer-Verlag, 1996, pp.15-25.

2. D.Bleichenbacher, Generating ElGamal signatures without knowing the secret, In Advances in Cryptology - EUROCRYPT'96, LNCS 1070, Springer-Verlag, 1996, pp.10-18.

3. M.Bellare and P.Rogaway, Random oracles are practical: a paradigm for designing efficient protocols, In Proc. of 1st ACM Conference on Computer and Communications Security, 1993, pp.62-73.

4. E.F.Brickell, Invited lecture given at Crypto'96, unpublished.

5. T.Elgamal, A public key cryptosystem and a signature scheme based on discrete logarithms, IEEE Trans. Inform. Theory, IT-31, 1985, pp.469-472.

6. Knuth, The Art of Computer Programming, Vol. 2, Addison-Wesley, 1981.

7. C.H.Lim and P.J.Lee, More flexible exponentiation with precomputation, In $A d-$ vances in Cryptology - CRYPTO'94, LNCS 839, Springer-Verlag, pp.95-107.

8. C.H.Lim and P.J.Lee, A key recovery attack on discrete log based schemes using a prime order subgroup, In Advances in Cryptology - CRYPTO'97, LNCS 1294, Springer-Verlag, pp.249-263.

9. C.H.Lim, H.S.Hwang and P.J.Lee, Fast modular reduction with precomputation, Proc. of 1997 Korea-Japan Joint Workshop on Information Security and Cryptology (JW-ISC'97), Oct. 26-28, 1997, pp.65-79.

10. M.Michels, D.Naccache and H.Petersen, GOST 34.10 - A brief overview of Russia's DSA, Computers and Security, 15(8), 1996, pp.725-732.

11. A.M.Odlyzko, The future of integer factorization, CryptoBytes, 1(2), 1995, pp.5-12.

12. P.C.van Oorschot and M.J.Wiener, On Diffie-Hellman key agreement with short exponents, In Advances in Cryptology - EUROCRYPT'96, LNCS 1070, SpringerVerlag, 1996, pp.332-343.

13. D.Pointcheval and J.Stern, Security proofs for signature schemes, In Advances in Cryptology - EUROCRYPT'96, LNCS 1070, Springer-Verlag, 1996, pp.387-398.

14. D.Pointcheval and S.Vaudenay, On provable security for digital signature algorithms, a manuscript, 1996, available from $h t t p: / / w w w . d m i . e n s . f r / \sim$ pointche/.

15. R.L.Rivest / M.E.Hellman / J.C.Anderson, Responses to NIST's proposal, Comm. ACM, 35(7), 1992, pp.41-52.

16. R.L.Rivest, A.Shamir and L.Adleman, A method for obtaining digital signatures and public key cryptosystems, Commun. ACM, 21(2), 1978, pp.120-126.

17. J.Stern, The validation of cryptographic algorithms, In Advances in Cryptology ASIACRYPT'96, LNCS 1163, Springer-Verlag, 1996, pp.301-310.

18. S.Vaudenay, Hidden collisions on DSS, In Advances in Cryptology - CRYPTO'96, LNCS 1109, Springer-Verlag, 1996, pp.83-88.

19. NIST, Digital signature standard, FIPS PUB 186, 1994. 


\section{A Domain Parameter Generation for KCDSA}

During the KCDSA initialization stage, a trusted authority in each domain have to generate and publish $p, q, g$ such that

- $p$ is a prime of specified length such that a prime $q$ of specified length divides $p-1$ and that all prime factors of $(p-1) / 2 q$ are greater than $q$.

$-g$ is a generator of a subgroup of $\mathbf{Z}_{p}^{*}$ of order $q$, i.e., $g$ is an element of $\mathbf{Z}_{p}$ such that $g^{q}=1 \bmod p$ and $g \neq 1$. Such a $g$ can be generated by testing $g^{(p-1) / q}=1 \bmod q$ with random $1<g<p$.

As an example, we describe a method for generating primes $p, q$ such that $(p-1) / 2 q$ is also prime. Let $P R G(s, n)$ denote a pseudorandom number generator on input $s$ generating an $n$-bit random number, defined by:

$$
\begin{aligned}
v_{i} & =h(s+i \bmod q) \text { for } i=0,1, \cdots, k-1, \\
v_{k} & =h(s+k \bmod q) \bmod 2^{r} \\
P R G(s, n) & =v_{k}\left\|v_{k-1}\right\| \cdots v_{1} \| v_{0}
\end{aligned}
$$

where $k=\frac{n}{L_{q}}$ and $r=n \bmod L_{q}$. The procedure for generating $p, q$ (of size $L_{p}, L_{q}$, respectively) and $g$ is as follows (see also Figure 1):

1. choose an arbitrary integer $s$ of at least $L_{q}$ bits.

2. initialize five counters: $t$ Count $=r$ Count $=1, p$ Count $=q$ Count $=$ $g$ Count $=0$.

3. form Seed for $P R G$ as:

$$
\begin{aligned}
w_{2} & =0 x 00\|i\| j \| t \text { Count, } \\
w_{1} & =r \text { Count } \| \text { pCount }, \\
w_{0} & =q \text { Count } \| \text { gCount } \| 0 x 00, \\
\text { Seed } & =s\left\|w_{2}\right\| w_{1} \| w_{0},
\end{aligned}
$$

where $i$ and $j$ are 8 bit numbers such that $L_{p}=512+256 i$ and $L_{q}=128+32 j$, tCount and gCount are 8 bits long, and $p$ Count, qCount and rCount are 16 bits long. It is assumed that Seed is automatically updated whenever any counter is changed.

4. generate a random number $r$ of length $L_{p}-L_{q}-1$ bits as follows:

$$
\begin{aligned}
& u=P R G\left(\text { Seed }, L_{p}-L_{q}-1\right), \\
& r=2^{L_{p}-L_{q}-2} \vee u \vee 1,
\end{aligned}
$$

where $\vee$ denotes bitwise-or.

5. test $r$ for primality (e.g., using the Miller-Rabin probabilistic primality test [6, page 379]). If $r$ is prime, go to step 8 .

6 . increment $r$ Count by 1 .

7. If $r$ Count $<2048$, go to step 4 . Otherwise, go to step 1 . 
8. set $p$ Count $=1$ and $q$ Count $=1$.

9. generate a random number $q$ of length $L_{q}$ bits using the updated Seed as follows:

$$
\begin{aligned}
& u=P R G\left(\text { Seed }, L_{q}\right), \\
& q=2^{L_{q}-1} \vee u \vee 1 .
\end{aligned}
$$

10. compute $p=2 q r+1$. If $|p|<L_{p}$, go to step 12 .

11. test $q$ for primality. If $q$ is prime, go to step 14 .

12. increment $q$ Count by 1 .

13. If $q$ Count $<1024$, go to step 9. Otherwise, go to step 15 .

14. test $p$ for primality. If $p$ is prime, go to step 19 .

15. increment $p$ Count by 1 and set $q$ Count $=1$.

16. If $p$ Count $<4096$, go to step 9 .

17. increment tCount by 1 .

18. If $t$ Count $<256$, go to step 3 . Otherwise, go to step 1 .

19. set $g$ Count $=1$.

20. generate a random number $u$ of length $L_{p}$ bits using the updated Seed as follows:

$$
u=P R G\left(\text { Seed, } L_{p}\right) .
$$

21. compute $g=u^{(p-1) / q} \bmod p$. If $g \neq 1$, go to step 24 .

22. increment gCount by 1.

23. If $g$ Count $<256$, go to step 20. Otherwise, go to step 17.7

24. terminate with output $p, q, g$ and Seed.

The Seed output can serve as a certificate for proper generation of the parameters $p, q$ and $g$. Anyone can check that $p, q$ and $g$ are generated as specified, since Seed contains all necessary information to verify their proper generation. For example, the following parameters $(|p|=1024,|q|=160)$ were generated using the described algorithm, where we the initial user input $s$ was taken as the first 160 bits of the fractional part of $\pi=3.14159 \cdots$ ¿ From the seed, we can see that $r=(p-1) / 2 q$ was found by testing 991 random numbers $(r$ Count $=0 x 3 d f=991)$ and $p$ was found by testing 1192 primes of $q$ ( $p$ Count $=0 x 77 c=1192$ ) and so on. It is easy to verify that these parameters are generated according to the above procedure.

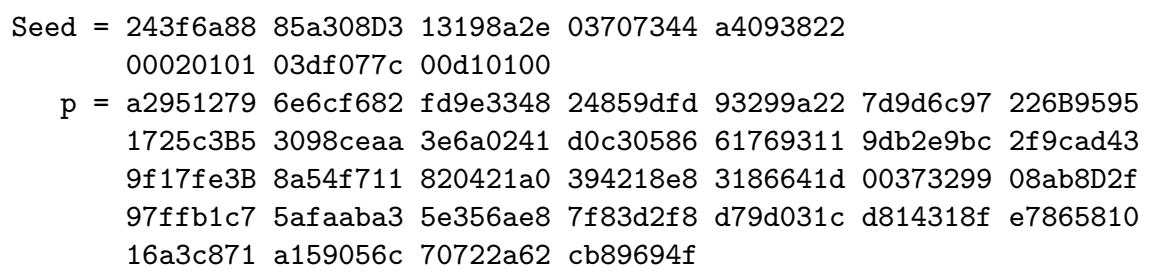

7 The probability of $g$ Count exceeding 255 is negligible ( $g$ Count $=1$ for almost all cases). For completeness, we simply make the control to go back to step 3 in such an exceptional case (through steps 17, 18, 3). 
$q=\operatorname{ada} 5 f f 8 f 174 c a b 840 c 846634$ dede6e81 5ac8f6ef

$g=1 b 2 f 2 d 3 b$ a6551ffd a74ca533 011f1a92 8277d572 67297496 78a42bda 5ba6c181 9cf283ee 14a3fb44 dacbe42b b9720d2d 7137c81e 69cfc7cf 20a41bb1 e117fa7d 9b8d0cb0 73a91e51 15c08db8 60be3633 67a08ac2 b59137c2 0ccf54b9 0dbc2c8c 90958555 d76c0020 2798282a 23cafc54 $7 \mathrm{c7e} 7820$ cf979902 2d3cde88 52d13753

\section{Conditions:}
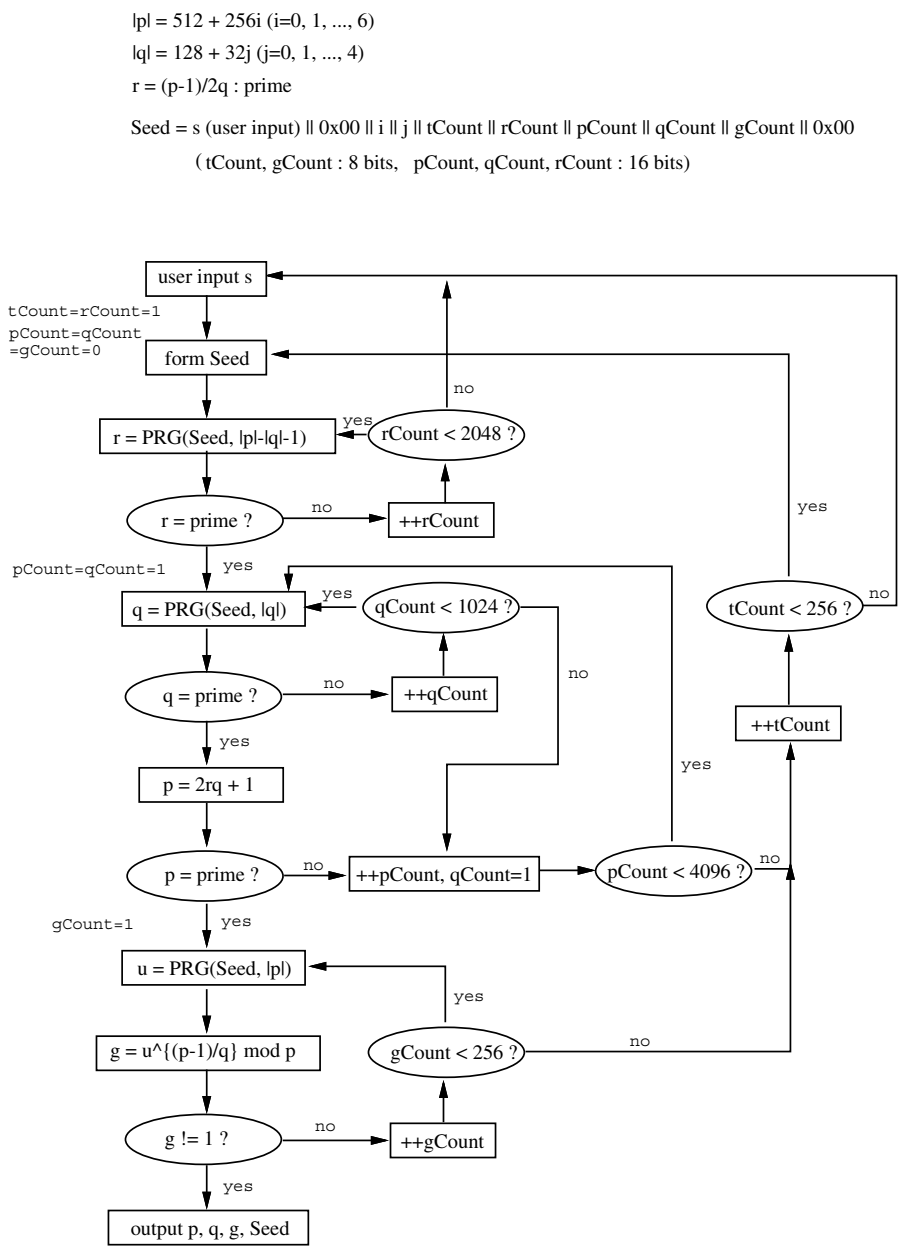

Fig. 1. Flow chart for generation of $p, q, g$ 